\title{
PRINCIPAL COMPONENT ANALYSIS BASED APPROACH FOR FAULT DIAGNOSIS IN PNEUMATIC VALVE USING DAMADICS BENCHMARK SIMULATOR
}

\author{
A.Kowsalya ${ }^{1}$, B.Kannapiran ${ }^{2}$ \\ ${ }^{I}$ M.Tech Student, Department of Instrumentation \& Control Engineering, Kalasalingam University, Tamilnadu, India \\ ${ }^{2}$ Professor\& Head, Department of Instrumentation \& Control Engineering, Kalasalingam University, Tamilnadu, \\ India
}

\begin{abstract}
This paper presents Artificial Neural Network (ANN) based classifier approach for fault diagnosis of pneumatic valve used in process industry. The proposed approach uses back propagation algorithm (BPN) to detect and diagnose the faults in pneumatic valve under normal and faulty operating conditions. Artificial Neural Network is trained using BPN algorithm to capture the relationship between the fault symptom and fault type. The required data for the development of ANN model were collected from the Development and Application of Methods for Actuator Diagnosis in Industrial Control Systems (DAMADICS) pneumatic valve simulator under normal and abnormal operating conditions. The performance of the proposed ANN model is improved by proposing suitable dimensionality reduction technique like Principal Component Analysis (PCA). The performance of the ANN model with PCA is compared with the performance of the ANN model without PCA. ANN model with PCA results in reduced mean square error during training and testing.
\end{abstract}

Keywords: Fault Diagnosis, Principal Component Analysis, Artificial Neural Network, Pneumatic Valve

\section{INTRODUCTION}

Pneumatic Valve is one of the vital components in process industry and it is necessary to monitor the condition of the pneumatic valve on a regular basis in order to prevent the pneumatic valve from damaged condition. The increasing complex process in industries and the necessity to reduce the cost for overall production, an automated approach is needed to detect and diagnose the various types of faults in pneumatic valve. Furthermore, by monitoring the condition of pneumatic valve, the maintenance strategy could be predicted. Also, the availability of the process plant will improve and the unpredictable faults which lead to production loss will also be reduced.

For fault diagnosis, it is important that the maintenance engineer should have prior knowledge about the pneumatic valve and its operation. The working of the pneumatic valve under normal and abnormal conditions is given by DAMADICS benchmark and the fault detection and identification approaches [10] have been identified. Many analytical methods such as state observer, parameter estimation and parity relation approach for fault diagnosis $[11,12]$ have been proposed. The issue with the analytical methods is that they do not provide the accurate model of the system. Later knowledge-based techniques were proposed which involves the knowledge acquired from the system structure description and operating conditions were used to develop the classical expert systems [13]. The main weakness of this technique is the bottleneck in knowledge acquisition and that the Boolean operators which are used for making binary logical decisions do not reflect the nature of many real world problems.

Later Fuzzy logic approach [5], [14] was introduced to define the behavior of the system using the set of rules. To define the set of rules for more number of variables is the disadvantage of the fuzzy logic technique. Many researchers proposed the ANN based method for fault diagnosis of pneumatic valve [4], [15, 16]. The artificial neural network has the capability of capturing the nonlinear relationship between the input variables \& output variables. Some researchers have developed ANN models for fault diagnostic to detect the problems rotating machine parts [17], Fisher Rosemount valves[18, 19], RCMAC [20]. ANN based method has been proposed for fault detection and identification of pneumatic valve using residuals from reference models [6]. The predictive fault detection and diagnosis technique has been presented with various artificial neural network algorithms in [2]. But the neural network approach needs enormous amount of data to develop the network model and the irrelevant variables in the input reduce the performance of the network. By selecting only the relevant variables as input features better performance is to be achieved with little computational effort. Therefore, effective methods for dimensionality reduction technique such as principal component analysis (PCA) have been developed. PCA [3] is a method based on the data projection into a low dimensional space that accurately characterizes the state of the system. PCA and ANN based approaches have been presented for online fault 
detection and classification of industrial machine simulator [7].

The paper is organized as follows: in the second section, the system description for this study is outlined. Section 3 describes the fault detection in pneumatic valve. Sections 4 describe the development of ANN model for fault diagnosis. Section 5 describes the Principal component analysis method and section 6 presents the results and discussion and finally, in Section 7, conclusions are drawn from the result.

\section{SYSTEM DESCRIPTION}

A common final control element in recent process industries is the pneumatic valve. The pneumatic valve is used for the manipulation of flow of gas, water, or chemical compounds, to compensate for the disturbance of load and it keeps the process variable regulated possibly close to the desired set point. The physical structure of the pneumatic valve is shown in figure 1 and the detailed description of the system is given in [2].

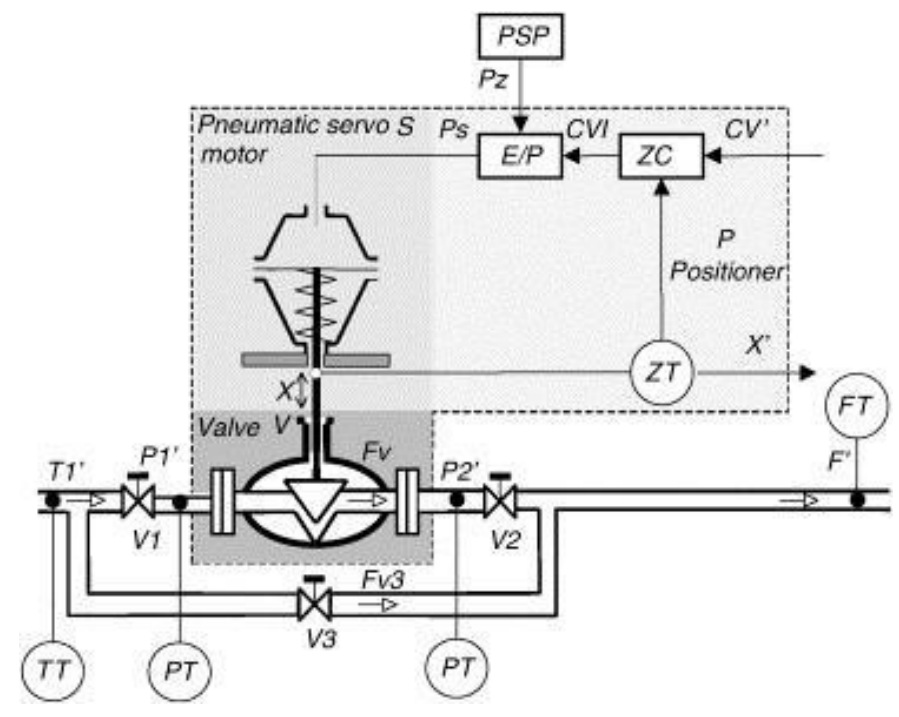

Fig-1: Physical Structure of Pneumatic Valve

The Pneumatic Valve consists of several sensors that measure the variables which influence the system, namely the upstream and downstream water pressures $(\mathrm{P})$, the water temperature $(\mathrm{T})$, the position of the $\operatorname{rod}(\mathrm{X})$, and the flow through the valve $(\mathrm{F})$. The three measured variables $\mathrm{P} 1, \mathrm{P} 2$, $\mathrm{T}$, along with $\mathrm{CV}$, can be taken as the inputs to the system while the latter two can be seen as its outputs. The description of the main parameters of the pneumatic valve is given in Table 1.

Table-1: Pneumatic Valve Parameters

\begin{tabular}{|l|l|}
\hline PSP & Position of Supply Air Pressure \\
\hline PT & Air Pressure Transmitter \\
\hline FT & Volume Flow Rate Transmitter \\
\hline TT & Temperature Transmitter \\
\hline
\end{tabular}

\begin{tabular}{|l|l|}
\hline ZT & Rod Position Transmitter \\
\hline E/P & Electro-Pneumatic Converter \\
\hline V1,V2 & Cut-off Valves \\
\hline V3 & By-Pass Valve \\
\hline Ps & $\begin{array}{l}\text { Pneumatic Servomotor Chamber } \\
\text { Pressure }\end{array}$ \\
\hline CVI & Controller Output \\
\hline CV & Control Reference Value \\
\hline F & Volumetric Flow \\
\hline $\mathrm{X}$ & Servo-Motor Rod Displacement \\
\hline
\end{tabular}

\section{FAULT DETECTION IN PNEUMATIC}

\section{VALVE}

Fault is defined as the malfunction or unexpected change of a parameter of the system from the pre-defined standard condition. Early detection of the fault may provide warning on evolving problems with appropriate actions taken to avoid serious process failures. Fault Diagnosis is the determination of the type of fault occurred, also location, time and magnitude of fault.

Development and Application of Methods for Actuator Diagnosis in Industrial Control Systems (DAMADICS) committee is concerning on the development of fault diagnosis algorithms. The DAMADICS [8, 9] data used for real time testing on industrial actuators done using various fault detection and diagnosis methods has been collected from Lublin Sugar Factory, Poland. The major goal of DAMADICS benchmark is the creation of well defined, repeatable single actuator faults. DAMADICS predefined the nineteen types of faults which may occur during the process in the pneumatic valve. The pneumatic valve faults are classified into four following groups. They are faults in control valve, pneumatic servo-motor, positioner and General faults/external faults. The nineteen numbers of faults in pneumatic valve are given with description in [2].

\subsection{Architecture of ANN}

The most commonly used architecture for ANN is Multilayer Feed Forward Neural Network (MLFFNN). The advantage of the artificial neural network technique is the generalization capability which deals with partial or noisy inputs. The $N$ component inputs, $x_{1}, x_{2} \ldots \ldots x_{N}$ are fed with weights $w_{1}, w_{2}$ $\ldots . . w_{N}$, and those outputs are summed in a proper way. An additional bias input with a weight is also applied. The multilayer feed-forward artificial neural network architecture shown is given in [4]. The network consists of three layers namely input, hidden and output layer. The input and output nodes are selected depending on the number of process parameters being considered. The non-linear mapping of the input and output variables is obtained by training the neural network using back propagation algorithm. 
In order to capture the non-linear relationship for the given input and output pairs, the weights are adjusted by training the network using back propagation algorithm. The network is trained using scaled conjugate gradient descent back propagation algorithm in which the weights and biases are moved in the opposite direction to the gradient of the error. Each following step of the gradient during learning gives a smaller and smaller error until a minimum error is reached.

\section{DEVELOPMENT OF ANN MODEL FOR}

\section{FAULT DIAGNOSIS}

The Artificial Neural Network with Principal Component Analysis is the proposed approach for fault diagnosis in pneumatic valve.

The development of ANN model consists of two phases namely training and testing. Figure 2 shows the schematic representation of the steps in ANN model development. The steps involved in the ANN model development using Back Propagation Algorithm are as follows,

\subsection{Input and Output Variables Selection}

The relationship between the input variables and output variables is learned by training the Artificial Neural Network. The input variables are chosen to represent the operating condition of the pneumatic valve. The normal and abnormal conditions of the pneumatic valve are represented by the output variables.

\subsection{Generation of Training Data}

The data is generated using DAMADICS pneumatic valve simulator. The MATLAB simulink environment is used to develop the DAMADICS simulator [9]. The data is collected for normal and faulty operating conditions of pneumatic valve. The training data is generated by considering the full range of operating conditions of the pneumatic valve, which contains all the possible fault occurrences.

\subsection{Data Normalization}

The data are normalized and presented to the neural network so that equal priority will be given to all the inputs. The normalization of data is done by compressing the range of training data between 0 and 1 using the expression,

$$
X_{n}=\frac{X-X_{\min }}{X_{\max }-X_{\min }}+\text { Starting Value }
$$

In Equation (1), the normalized value of the data is represented by $X_{n}$. The minimum value and maximum values among all the values of the data is represented by $X_{\min }$ and $X_{\max }$.

\subsection{Training and Testing of Network}

The back propagation algorithm is used to train the neural network with the training dataset. Followed by training, the network is tested with the test dataset to assess the performance of the developed network.

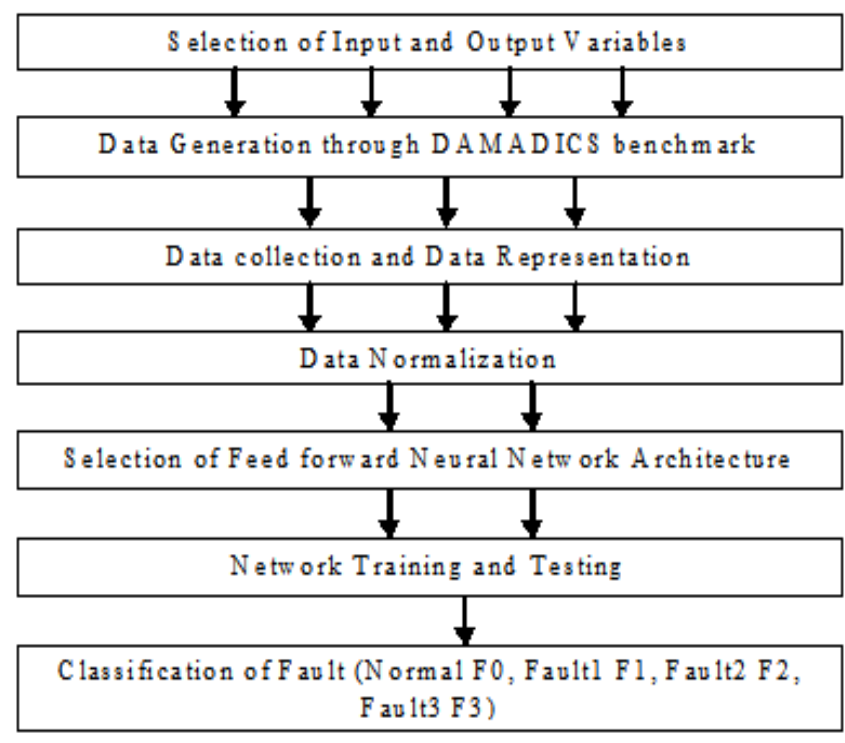

Fig-2: Schematic Representation of ANN Model for Fault Diagnosis

\section{PRINCIPAL COMPONENT ANALYSIS}

Principal component analysis (PCA) is one of the feature extraction techniques. This dimensionality reduction technique is used abundantly in all forms of analysis from neuroscience to computer graphics because it is a simple method of extracting only the relevant information from large data sets. With smaller effort, PCA provides a roadmap for how to reduce a complex data set to a lower dimension. The main idea of this technique is to be able to compute the most relevant components or factors from the original features.

\subsection{Procedure for PCA}

Reduction in dimensionality can be achieved either by eliminating data closely related with remaining data in the available dataset, or data is combined to form smaller set of features. Using the example of projecting data from two dimensions to one, a linear projection requires the optimum choice of projection to be a minimization of the sum-ofsquares error. The sum of squares error is obtained first by subtracting the mean $\mathrm{x}$ of the data set. The Eigenvectors and Eigen-values of the covariance matrix are calculated. The eigenvectors corresponding to the $\mathrm{M}$ largest Eigen-values are retained, and the input vectors $\mathrm{x}^{\mathrm{n}}$ are subsequently projected onto the eigenvectors to give components of the transformed vectors $\mathrm{z}^{\mathrm{n}}$ in the $\mathrm{M}$ - dimensional space. Retaining a subset $\mathrm{M}<\mathrm{d}$ of the basis vectors $\mu_{\mathrm{i}}$ so that only M coefficients $z_{\mathrm{i}}$ used allows for replacement of the remaining coefficients by constants $b_{i}$. This replacement allows each $\mathrm{x}$ vector to be approximated by equation (2)

$$
\bar{x}=\sum_{i-1}^{m} z_{i} \mu_{i}+\sum_{1-m+1}^{d} b_{i} \mu_{i}
$$

Where in equation (2), $\mu_{i}$ represents a linear combination of $d$ orthonormal vectors 


\section{RESULTS \& DISCUSSION}

In this paper the data is collected using the DAMADICS pneumatic valve simulator [1] and the ANN model is developed for fault detection. Totally 1000 data was generated under normal and abnormal operating condition of pneumatic valve. The data was divided into two sets randomly, 750 data used for training and 250 data used for testing the performance of the network. The input variables along with their range are given in the Table 2. The output variables along with class are given in Table 3.

Table-2: Input Variables with Range

\begin{tabular}{|l|l|l|}
\hline $\begin{array}{l}\text { Input } \\
\text { Variable } \\
\text { Code }\end{array}$ & $\begin{array}{l}\text { Name of the Input } \\
\text { Variable }\end{array}$ & $\begin{array}{l}\text { Range with } \\
\text { Units }\end{array}$ \\
\hline $\mathrm{CV}$ & $\begin{array}{l}\text { Control Valve } \\
\text { (Controller Output) }\end{array}$ & $0-100(\%)$ \\
\hline $\mathrm{P} 1$ & $\begin{array}{l}\text { Water Pressure } \\
\text { (Valve Inlet) }\end{array}$ & $0-4000(\mathrm{KPa})$ \\
\hline $\mathrm{P} 2$ & $\begin{array}{l}\text { Water Pressure } \\
\text { (Valve Outlet) }\end{array}$ & $0-4000(\mathrm{KPa})$ \\
\hline $\mathrm{T}$ & $\begin{array}{l}\text { Water Temperature } \\
\text { (Valve Outlet) }\end{array}$ & $0-150(\mathrm{deg} \mathrm{C})$ \\
\hline
\end{tabular}

Table-3: Output Variables along with Class

\begin{tabular}{|l|l|l|}
\hline $\begin{array}{l}\text { Name of the Output } \\
\text { Variable }\end{array}$ & Class \\
\hline Normal (No Fault) & 0 & 0 \\
\hline Fault 1 (Valve Clogging) & 0 & 1 \\
\hline $\begin{array}{l}\text { Fault 2 (Valve Plug or Valve } \\
\text { seat sedimentation) }\end{array}$ & 1 & 0 \\
\hline $\begin{array}{l}\text { Fault 3 (Internal Leakage } \\
\text { Valve Tightness) }\end{array}$ & 1 & 1 \\
\hline
\end{tabular}

Table-4: ANN Model Performance with PCA

\begin{tabular}{|l|l|}
\hline Performance Measures & Performance Values \\
\hline Number of Training Data & 750 \\
\hline Number of Testing Data & 250 \\
\hline Mean Square Error for Training & 0.0050 \\
\hline Mean Square Error for Testing & 0.0036 \\
\hline Training Time (sec) & 26.6918 \\
\hline $\begin{array}{l}\text { Number of data correctly } \\
\text { classified }\end{array}$ & 249 \\
\hline$\%$ of Fault Classified & 99.6 \\
\hline
\end{tabular}

The data are normalized and this is used for training the Artificial Neural Network with Principal component analysis. Dimensionality reduction is done in the input data. Then the data is given to ANN model after dimensionality reduction. The ANN model with PCA was trained using BPN algorithm and scaled conjugate gradient is used as the training function. The network generalization performance is evaluated with 250 test data after training. Considering ANN with PCA, for 13 hidden layers, MSE for training is 0.0050 and MSE for testing is 0.0036 . The training time taken by the network is 26.6918 seconds. Out of 250 test data, 249 data were correctly classified and the percentage of fault classification achieved is 99.6. The performance of ANN model with PCA is given in the Table 4.

Figure 3 shows the training performance of ANN model with PCA. For comparison, the ANN model is considered without Principal Component Analysis. The input data was given to the multi layer feed forward neural network without dimensionality reduction. Considering ANN model without PCA, for 9 hidden layers, MSE for training is 0.0053 and MSE for testing is 0.0045 . The training time taken by the network is 24.6794 seconds. Out of 250 test data, 248 data were correctly classified and the percentage of fault classification achieved is 99.2. When comparing the performance of the ANN model with PCA and ANN model without PCA, the mean square error for training \& testing was low and also the percentage of fault classification was improved and this is given in Table 5. This highlights that the ANN with PCA shows improved performance when compared with ANN without PCA.

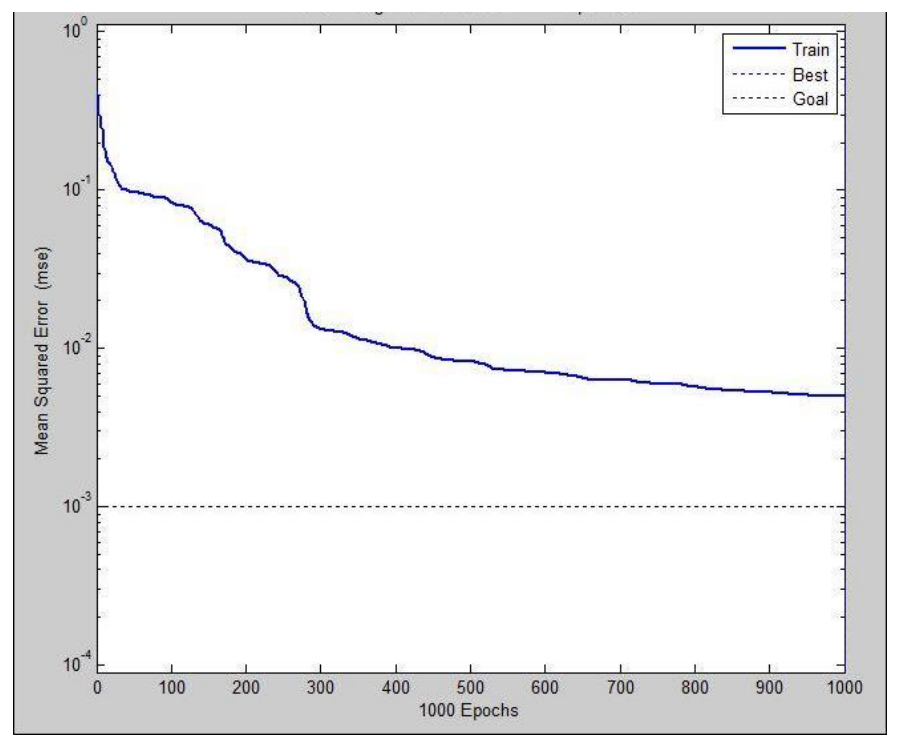

Fig-3: Training Performance of ANN Model with PCA

Table-5: Performance Comparison of ANN Model with \& without PCA

\begin{tabular}{|l|l|l|}
\hline Description of Parameters & $\begin{array}{l}\text { ANN with } \\
\text { PCA }\end{array}$ & $\begin{array}{l}\text { ANN } \\
\text { without } \\
\text { PCA }\end{array}$ \\
\hline Number of Training Data & 750 & 750 \\
\hline Number of Testing Data & 250 & 250 \\
\hline Mean Square Error for Training & 0.0050 & 0.0053 \\
\hline Mean Square Error for Testing & 0.0036 & 0.0045 \\
\hline Training Time(sec) & 26.6918 & 24.6794 \\
\hline Number of data correctly & 249 & 248 \\
\hline
\end{tabular}




\begin{tabular}{|l|l|l|}
\hline classified & & \\
\hline \% of Fault Classified & 99.6 & 99.2 \\
\hline
\end{tabular}

Next, the performance of the ANN model is tested under noisy condition. The ANN model is developed with PCA and without PCA under noisy condition and is given in Table 6. Considering ANN model with PCA under noisy condition, for 13 hidden layers, MSE for training is 0.0051 and MSE for testing is 0.0027 . The training time taken by the network is 27.0506 seconds. Out of 250 test data, 249 data were correctly classified and the percentage of fault classification achieved is 99.6. On comparing the performance of the ANN model with PCA and ANN model without PCA under noisy conditions, the mean square error for training \& testing was low. The obtained results given in Table 6 shows that the ANN model with PCA gives improved performance under noisy conditions.

Table-6: Performance of ANN model with \& without PCA under noisy condition

\begin{tabular}{|l|l|l|}
\hline Description of Parameters & $\begin{array}{l}\text { ANN with } \\
\text { PCA }\end{array}$ & $\begin{array}{l}\text { ANN } \\
\text { without } \\
\text { PCA }\end{array}$ \\
\hline Number of Training Data & 750 & 750 \\
\hline Number of Testing Data & 250 & 250 \\
\hline Mean Square Error for Training & 0.0051 & 0.0053 \\
\hline Mean Square Error for Testing & 0.0027 & 0.0032 \\
\hline Training Time(sec) correctly & 27.0506 & 25.6778 \\
\hline $\begin{array}{l}\text { Number of data } \\
\text { classified }\end{array}$ & 249 & 249 \\
\hline \% of Fault Classified & 99.6 & 99.6 \\
\hline
\end{tabular}

\section{CONCLUSIONS}

This paper presents the artificial neural network (ANN) model for fault diagnosis of pneumatic valve. The ANN model was developed using the data generated under normal and faulty conditions of Pneumatic Valve using the DAMADICS benchmark simulator. The performance of the ANN model has improved by proposing suitable dimensionality reduction technique like Principal Component Analysis. The mean square error for training and testing is reduced and also the fault classification accuracy is high using ANN model with PCA. The performance of the model is evaluated under noisy condition and the reduced mean square error for training and testing is achieved and the accuracy of classification is also improved using ANN model with PCA. This shows that the effectiveness of the ANN model with PCA shows improved performance when compared with ANN model without PCA. The classification accuracy of the developed ANN model can be further improved, by proposing Adaptive Neuro-Fuzzy Inference System (ANFIS) model for further research work.

\section{REFERENCES}

[1]. DAMADICS 2000, Research training network damadicsproject,

http://eng.hull.ac.uk/research/control/damadics.html
[2]. Sundarmahesh.R, Kannapiran.B, "Fault Diagnosis of Pneumatic Valve with DAMADICS Simulator using ANN based Classifier Approach", International Journal of Computer Applications (0975-8887), 2013

[3]. P.Subbaraj, B.Kannapiran, "Fault Diagnosis of Pneumatic Valve using PCA and ANN techniques", CCSEIT, CCIS 204, pp 404-413, 2011, Copyright SpringerVerlag Berlin Heidelberg 2011

[4]. P.Subbaraj, B.Kannapiran, “Artificial Neural Network Approach for Fault Detection in Pneumatic Valve in Cooler Water Spray System", International Journal of Computer Applications (0975-8887), Volume 9-No.7, November 2010 [5]. P. Subbaraj, B.Kannapiran, "Fuzzy based fault detection and diagnosis in pneumatic actuator in cement industry", $P$. Subbaraj et. al. / International Journal of Engineering Science and Technology, Vol. 2(9), 4095-4107, 2010

[6]. Kourd Y, Leebvre D, Guersi N, "Early FDI based on residuals design according to the analysis of models of faults", Advances in Artificial Neural Systems, Article ID 453169, 1-10, 2011

[7]. Zhou JH, Pang CK, Lewis FL, Zhong ZW, "Dominant Feature Identification for Industrial Fault Detection and Isolation Applications", Expert Systems with Applications, Vol.38, 10676-10684, 2011

[8]. Binoy B. Nair , “A Novel Feature Selection method for Fault Detection and Diagnosis of Control Valves", IJCSI(8), pp.415-419, 2011

[9]. Tarun Chopra, Jayashri Vajpai., "Fault Diagnosis in Benchmark Process Control System Using Stochastic Gradient Boosted Decision Trees", IJSCE(1), pp.98-101, 2011

[10]. M.Blanke and R.J.Patton, "Industrial Actuator Benchmark for Fault Detection and Isolation", ControlEng. Practice, Vol. 3, No. 12, pp. 1727-1730, Copyright (C) 1995 Elsevier Science Ltd, 1995

[11]. R.Isermann, "Supervision, Fault-Detection and FaultDiagnosis Methods - An Introduction", Control Eng. Practice, Vol. 5, No. 5, pp. 639-652, 1997

[12]. R.Isermann and P.Balle, "Trends in the application of Model-based Fault Detection and Diagnosis of Technical Processes", Control Eng. Practice. Vol. 5, No. 5, pp. 709719, 1997

[13]. S. Leonhardt and M. Ayoubi, "Methods of Fault Diagnsis", Control Eng. Practice, Vol. 5, No. 5, pp. 683692, Copyright (C) 1997 Elsevier Science Ltd, 1997

[14]. L.F. Mendonca, J.M.C. Sousa, J.M.G. Sa' da Costa, "An architecture for fault detection and isolation based on fuzzy methods", Expert Systems with Applications 36,1092-1104, 2009

[15]. M. Demetgul, I.N. Tansel, S. Taskin, "Fault diagnosis of pneumatic systems with artificial neural network algorithms", Expert Systems with Applications 36,1051210519, Elsevier, 2009

[16]. Joseph McGhee, Ian A. Henderson, Alistair Baird, "Neural networks applied for the identification and fault diagnosis of process valves and actuators", Measurement Vol. 20, No. 4, pp. 267-275,(C 1997 Elsevier Science Limited, 1997

[17]. S. Rajakarunakaran, P. Venkumar, D. Devaraj , K. Surya Prakasa Rao, "Artificial neural network approach for 
fault detection in rotary system", Applied Soft Computing 8, 740-748, 2008

[18]. M.Karpenko, N.Sepheri, "Neural Network classifiers applied to condition monitoring of a pneumatic process valve actuator", Engineering Applications of Artificial Intelligence 15, 273-283, 2002

[19]. M.Karpenko, N.Sepheri, D.Scuse, "Diagnosis of process valve actuator faults using a multilayer neural network", Control Engineering Practice 11, 1289-1299, 2003

[20]. Shengwei Wang, Zhiming Jiang, "Valve fault detection and diagnosis based on CMAC neural networks", Energy and Buildings 36, 599-610, Elsevier 2004 